\title{
Critical Node Detection in Large Scale Mobile Ad hoc Networks
}

\author{
Majid Ahmad \\ Faculty of Computer Science \\ and System Studies, \\ Mewar University, \\ Chittorgarh, India
}

\author{
Durgesh Kumar Mishra \\ Professor and Head \\ Department of Computer \\ Science Engineering, \\ Aurobindo Institute of \\ Technology, Indore, India
}

\begin{abstract}
Reliability calculation of large scale MANETs is an NP computational problem, and this complexity can be reduced by identifying critical nodes in a network. The identification of critical nodes itself is a computationally hard problem. The present work provides an empirical Algorithm for detecting critical nodes in a MANET which is computationally efficient than the already existing detection methods. The algorithm proposed for critical node detection is based on the pattern a critical node may exhibit uniquely in a connection matrix.
\end{abstract}

\section{General Terms}

Mobile ad-hoc networks, Critical Node, Reliability, and Network Scalability.

\section{Keywords}

Critical Node Detection, Large scale Mobile Networks, Network Reliability

\section{INTRODUCTION}

The era of mobile wireless technology seems to be getting matured with the leaps taken by researchers and industry in almost all the dimensions of wireless technology domain. The concerns of wireless technology ranging from bandwidth limitations to device miniaturization have been dealt. The evolution of wireless technology is noteworthy in general as a consequence of repeated tuning and adaptation of various techniques and technologies. The latest phases of this wireless evolution have made wireless technology quite exciting and vibrant. The core infrastructure has been developed in accordance to applications and scenarios. The applications of wireless have been around for a long and their categories have been thoroughly analyzed and developed. Some generic issues related to all the categories of wireless communication networks are there which not only have been dealt with in past, rather have been systematically investigated and remedied. The issue of fault tolerance is one of its kinds, which is more generic across the boundaries of different branches of science and engineering. The area of fault tolerance in computer networks in particular has a long history of adaptation and amendment. Wireless communication networks are no apart from its parent engineering branch of computer networks. Wireless network as evident from their behavior and cultivate are always more prone to faults of myriad reasons, thus making availability of wireless communication systems a concern of vital degree. Moreover wireless systems generally have applications which are more environment sensitive like the case of wireless sensor networks. The availability of wireless communication has been a concern from long, on one end it's being taken cared of while on the other end more issues are emerging to be fixed. The network breakdowns due to weather, shadowing, mobility, fading, intrusions and more are few of the important reasons questioning the availability of wireless networks. Mobile ad-hoc networks are inherently a class of wireless communication systems requiring more advanced availability measures. Mobile ad-hoc networks as having distributed control mechanism require each component to function so as to achieve over all connectivity. The reliability calculations of MANETs have been a concern due to their so called on the fly connectivity means. The dynamic configuration changes have always made reliability calculations in MANETs more challenging and time complex. The complexity is not only proportional to frequent mobility but also due to the stretching capability. A MANET may start spanning from few meters to few thousand meters or more as a consequence of mobility and node additions. Our previous work in this area has made a breakthrough by devising an algorithm for calculating reliability of large scale MANETs. The algorithm provides a realistic technique for calculating source to destination reliability taking into consideration both factors of mobility (dynamic configurations) and scale (size) [1]. The algorithm drastically reduces the average complexity (time) of calculating the reliability. The only assumption in the algorithm had been the critical node detection that was inherited form previous work of few authors. The present work provides a simplified technique for critical node detection. The algorithm proves to be realistic and more efficient than previous critical detection techniques.

\section{RELATED WORK}

All The past research in the area of critical node detection has been due to several reasons. The identification of critical node/links is a vital step in reducing the vulnerability of a network (wire-line/wireless), as these links can be a target for breaking a network down. The critical node detection also has also been used in routing algorithms for data redirection and all. A critical node detection may also be used in order to sub divide a network (decomposition). The techniques of detecting critical nodes in a network can be mainly categorized into two subcategories. The techniques which work on physical details of all the network nodes, thus requiring the pre knowledge of actual node coordinates. This category is quite unrealistic for large scale networks as reliability calculations are to be done from a remote location at least from majority of nodes and the coordinates may get disturbed by slight moment of wireless nodes. The second category of techniques involved in critical node detection are the techniques which does not require actual physical details of the network nodes rather, provide identification results based on the instant configuration of a network, so only requiring details of connectivity(adjacency matrix). The concept of critical nodes and there detection in wireless networks has been studied for reasons such as connectivity and communication and proves to be an effective measure [2] [3] [4]. The detection algorithms provided in past approaches 
to critical node detection were intended for connectivity concerns and vulnerability. The study of large scale wireless mobile ad hoc networks is a computationally intricate exercise and to deal with this problem few decomposition techniques have been devised which are quite helpful in better understanding these networks in a disintegrating manner [5] [6]. The models for network reliability whether stochastic or combinatorial have a long history of refinement and advancements. The measures in combinatorial approaches like RBD, fault-tress and reliability graphs seems to be more analytically sound and feasible than their counterparts of stochastic models. The study of wireless network reliability through reliability graphs somewhat maps more closely with the wireless scenarios. Moreover measures whether based on stochastic or combinatorial have been devised in order to take precise measures of availability and survivability. These measures provide us very accurate results about reliability of wireless networks, but they all inherited a limitation of growth. As the reliability calculation of wireless networks in general is a computationally hard problem, reliability analysis based on network decomposition seems to be the only means in evaluating the network reliability in a time bound manner .The most widely accepted and discussed technique for reliability calculation has been based on the concept of cuts (in graph) and that proves to be the only fundamental bedrock for advanced reliability analysis for all complex networks (wired/wireless) [7]. The minimal cut-sets and other related approaches for determining the reliability measures have been with us for quite a long [8] [9] [11]. The reliability evaluation schemes are generally categorized into few fundamental classes. The all-terminal-reliability reliability, source-terminal reliability and many-source- terminal reliability techniques. The k-terminal reliability measure as evident better maps with the network communication scenarios. The k-terminal reliability technique is a generalization of source-terminal reliability measure [12][13]. The wireless mobile ad-hoc networks are very closely mapped by two-terminal (sourceterminal) reliability measure [14] [15][16].

\section{PROBLEM DEFINITION}

A mobile ad hoc network as usually realized as an undirected graph $\mathrm{G}(\mathrm{V}, \mathrm{E})$ comprising of mobile wireless nodes (stations) denoted as ' $v$ ' and a total number of connections(Links) denoted as ' $E$ '. The number of links may be further categorized into connected and unconnected edges (links). The summation of the number of connected and unconnected links will always remain constant, while their individual values may vary as a function of time. Lets state the two categories as $\mathrm{Ec}$ and $\mathrm{Eu}$, implying that $\mathrm{E}=\mathrm{Ec}+\mathrm{Eu}$. $\mathrm{A}$ network in total can have $\mathrm{e}=\mathrm{V}(\mathrm{V}-1) / 2$ number of edges. The reliability of existence of a particular network configuration can be calculated in the following expression.

$$
\begin{aligned}
& \mathrm{R}_{\mathrm{ex}}= \\
& \quad \mathrm{e} \\
& \prod_{1}\left(\mathrm{NLN}_{\text {Prob }}\right)^{\mathrm{lt}}\left(1-\mathrm{NLN}_{\text {prob }}\right)^{1-\mathrm{lt}} \\
& 1
\end{aligned}
$$

The sum of all configuration probabilities

$\mathrm{S}_{\mathrm{Rex}}=\sum \mathrm{R}_{\mathrm{ex}}$ 1

Calculating the overall 2-terminal reliability of the network for any two nodes (Sender, Receiver) is as

$$
2^{\mathrm{e}}
$$

$2 \mathrm{TR} \mathrm{SR}=\sum \mathrm{S}_{\mathrm{Rex}} \mathrm{Pi}$

(Where $\mathrm{Pi}=1$ if at least a single path exists between selected sender and receiver otherwise $\mathrm{Pi}=0$ ).

As Evident from the above two terminal reliability calculation formulae, the complexity of reliability calculation is dependent on number of nodes in a network. The above fundamental approach of two terminal reliability calculations still stands to be one of the best reliability evaluations techniques till date at least for MANETs. The complexity of the calculation is thus exponentially proportional to the number ' $\mathrm{V}$ ' of the graph $\mathrm{G}(\mathrm{V}, \mathrm{E})$. The complexity of reliability calculation can be reduced only by limiting the number of configuration. The reliability calculation model provided in our previous work [1] has decomposed the problem into sub-problems and then calculated the two terminal reliability of a whole network. The decomposition was done on the basis of critical nodes (Links). A critical link divides a network into further networks (sub-networks) of smaller scale. The division of a network into a number of sub networks thus reduces the complexity radically. The critical link as defined is the link which provide only means of communication between different parts of a network (The removal of the link will disconnect the communication from source to receiver). The identification of critical links in a network is a prime step in reducing the reliability calculation for a large scale network. The past techniques used for this purpose had limitations of complexity and applicability. The techniques were more suitable to small scale MANETs, as increase in node number affected the complexity of critical node detection deficiently. The technique presented here is a simplified scheme for detecting a critical node in a large scale MANET with a controlled complexity as compared to till date existing schemes.

\subsection{Algorithm Introduction}

The algorithm proposed to find out critical nodes in an MANET is based on the second category of techniques where actual positions of nodes isn't a prerequisite rather node connectivity (link exists or not) is taken as input. This input has an added advantage over the others (actual node coordinates) as connectivity is more deterministic in a mobile environment rather than coordinate positions. Nodes are expected to be mobile and the frequency of mobility is inherently more rapid than link makeup and breakup. The input for node connectivity information can be best 
represented in terms of an adjacency matrix and this matrix gets more simplified as links are bidirectional in MANETs. The algorithm proposed for critical node detection is based on the pattern a critical node may exhibit uniquely in an adjacency matrix. The reliability calculator algorithm proposed in our previous work [1] is used for calculating final two terminal of the network. A Mobile ad-hoc network can be decomposed by expecting that critical nodes are present with some probability

\subsection{Algorithm for critical node detection}

Step1. Draw the adjacency Matrix of the network.

Step2. Test out whether the source \& Destination nodes (Columns) have single One: Declare them as critical nodes.

Step3. Test out how many connected node pairs have alternate corresponding values or zero-zero (column).

Step4. Select each such pair: remove this pair from the matrix: Find if a path still exists between Source \& Destination: If path exists (Node pair not critical) else (Node pair Critical).

\subsection{Algorithm Description}

The algorithm above algorithm decomposes a network into smaller sub networks based on the position of the critical nodes. The algorithm makes an efficient attempt to reduce the final reliability calculation by generating a fragmented outlook of a complex network. The algorithm works by primarily drawing an adjacency matrix of the whole network. A special case for source and destination nodes is to be verified to detect if they form critical nodes. The step3 onwards is the actual working of critical node detection. Only those node pairs are to checked who at the first place are connected to each other and also do not have any corresponding row with values (1-1). This selection makes this algorithm efficient as only a subset of node pairs are taken for calculation. Thus only those connected node pairs can be candidates for critical nodes who satisfy the previous condition. These probable critical node pairs are finally tested by removing them from the matrix and checking whether a path still exists from source to destination. An illustration of the above algorithm is presented in the next sub-section.

\subsection{Algorithm illustration}

The figure1 depicts a network $\mathrm{G}(\mathrm{V}=6, \mathrm{E})$ given and for reducing the computational complexity the network is to be divided into sub networks (if possible). The reduction is possible only if we can detect few critical nodes in the given network by a computationally efficient algorithm. The proposed scheme in the above section can be used to find a critical node. The node S1 (SN1) is the source node in this following network, while R6 is the destination node. The other nodes are the intermediate nodes, which may form or reform the links. The critical node at any moment of time (if present) can reduce the calculation complexity and will lead to an improved reliability measurement.

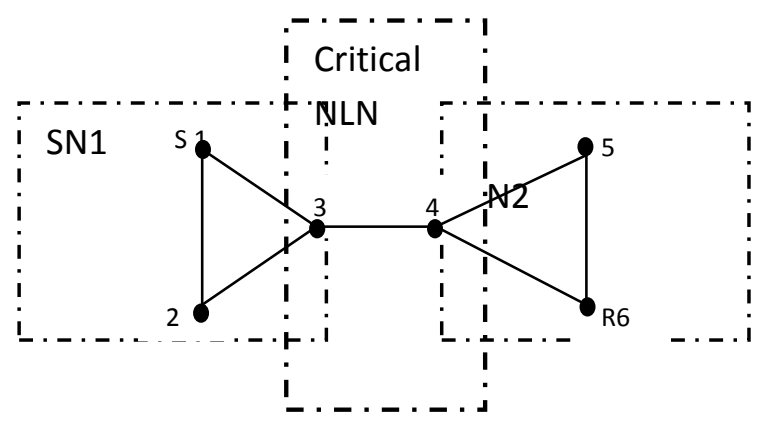

Figure 1: A Mobile Ad-hoc Network

The adjacency matrix for the above network is drawn as under.

\begin{tabular}{lll|ll|ll} 
& 1 & 2 & 3 & 4 & 5 & 6 \\
1 & 0 & 1 & 1 & 0 & 0 & 0 \\
2 & 1 & 0 & 1 & 0 & 0 & 0 \\
3 & 1 & 1 & 0 & 1 & 0 & 0 \\
4 & 0 & 0 & 1 & 0 & 1 & 1 \\
5 & 0 & 0 & 0 & 1 & 0 & 1 \\
6 & 0 & 0 & 0 & 1 & 1 & 0
\end{tabular}

As apparent both source and destination columns (Column number one \& six) in this adjacency matrix have more single value of one, thus they don't qualify for critical nodes. Further let's enumerate all the connected node pairs whose columns don't contain values (one one) for the same row. The enumeration will result in to the only pair for this example $\{(3,4)\}$. Taking each such node pair (only single in this case) from the adjacency matrix and if not detecting a source to destination path will qualify the node pair to be critical. For node pair $(3,4)$, the resultant matrix is reduced to

$\begin{array}{ccccc} & 1 & 2 & 5 & 6 \\ 1 & 0 & 0 & 0 & 0 \\ 2 & 1 & 0 & 0 & 0 \\ 5 & 0 & 1 & 0 & 1 \\ 6 & 0 & 1 & 1 & 0 .\end{array}$

The above adjacency matrix does not have a path form source to destination thus the node pair $(3,4)$ form a critical link. Thus this algorithm reduces the complexity of critical node identification by limiting the calculation. The selections of only those node pairs which qualify the condition at step 3 in the algorithm are only further verified. 


\section{Algorithm critical_link(G,n,source,destination)}

Is critical link: $=$ false

source_critical :=false;

destination_critical:=false;

If (there exist only one node $\mathrm{u}$ adjacent from source) /* check

for source node as critical-node

\{

Divide the network into single subnetwork G1;

critical_link(G1,n-1,u,destination);

source_critical =true;

\}

If (there exist only one node $\mathrm{v}$ adjacent from destination)/* check for source node as critical-node

\{

Divide the network into single subnetwork G1;

critical_link(G1,n-1,source,v);

destination_critical =true

For (each connected_link_pair (u,v) in G) do / * u can't be source node if source_critical =true , $v$ can't be destination node if destination_critical $=$ true) $* /$

If ( $\mathrm{u}, \mathrm{v}$ has alternate or zero-zero values at same column) then

If (by removing $(\mathrm{u}, \mathrm{v})$ link from $\mathrm{G}$ does not contain the path from source to destination) then

$$
\{
$$$$
\text { Is_critical_link := true; }
$$$$
\text { Break; }
$$$$
\text { \} }
$$$$
\text { \} }
$$

\}

if(is_critical_link=true)

\{

Divide the graph into two subnetworks $(\mathrm{G} 1, \mathrm{n} 1)$ and $(\mathrm{G} 2, \mathrm{n} 2)$ critical_link(G1,n1,source,u);

critical_link(G2,n2,v,destination);

\}

The above piece of pseudo-code results in calculating two terminal reliability by detecting critical nodes in a network and thus decomposing the network into different subnetworks resulting in complexity reduction. The complexity of the above proposed algorithm will always will have an upper bound $\mathrm{O}\left(\mathrm{n}^{2}\right)$. The reliability calculation complexity will significantly get reduced as this technique is embedded.

\section{RESULT SET}

The simulation results drawn for the proposed algorithm showed the complexity improvements over the existing critical node detection algorithms. Figure 2 shows us the comparison of growth for the existing and proposed method. The existence of critical nodes in a network was simulated on a standard topology known as Waxman random network topology. The topology generation is depicted in figure 3 , as this generation is for large scale mobile ad-hoc networks. The presence of critical nodes in randomly generated topologies was analyzed in figure4. The Simulation results of the proposed algorithm can be seen in the figure5.

\section{CONCLUSION}

The presence of critical nodes in a large scale wireless mobile ad-hoc network is being exploited in this work for decomposing a network. This decomposition in turn proves phenomenal in reducing the complexity of calculating the reliability of large scale networks in a time bound (computationally less complex) manner. An efficient algorithm for detection of these critical nodes was provided in this manuscript that proves to be very robust and realistic.

\section{ACKNOWLEDGEMENT}

Durgesh Kumar Mishra has done $\mathrm{PhD}$ (Computer Engineering). He is a senior member of IEEE.He is having around 21 Yrs of teaching experience and more than 6 Yrs of research experience. Presently he is working as Professor (CSE) and Dean (R\&D) in Sri Aurobindo Institute of Technology, Indore, India. He is also a Visiting Professor at IIT Indore.

Majid Ahmad is a $\mathrm{PhD}$ scholar, working in the area of MANET reliability. His past research has been in the area of MANET protocol tuning. He has four years of Research \& Teaching experience.

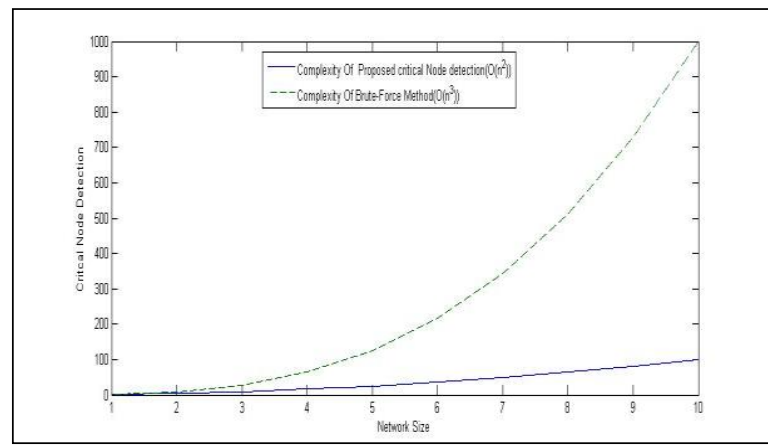

Figure 2: Proposed Critical Node Detection Algorithm

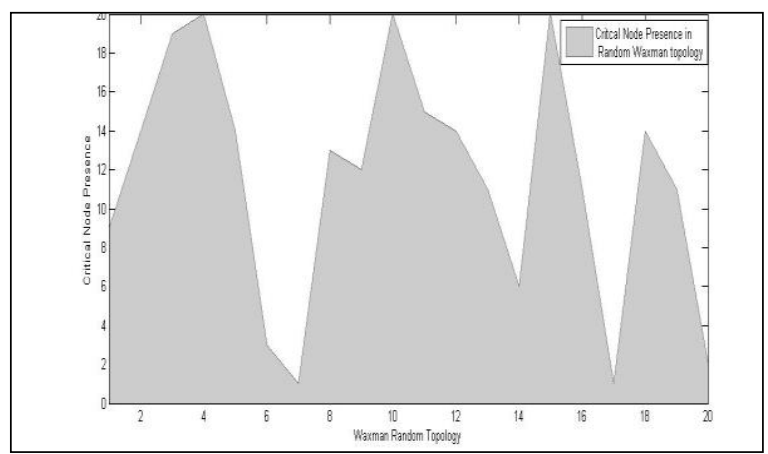

Figure 3: Waxman Random Network Topology Generation

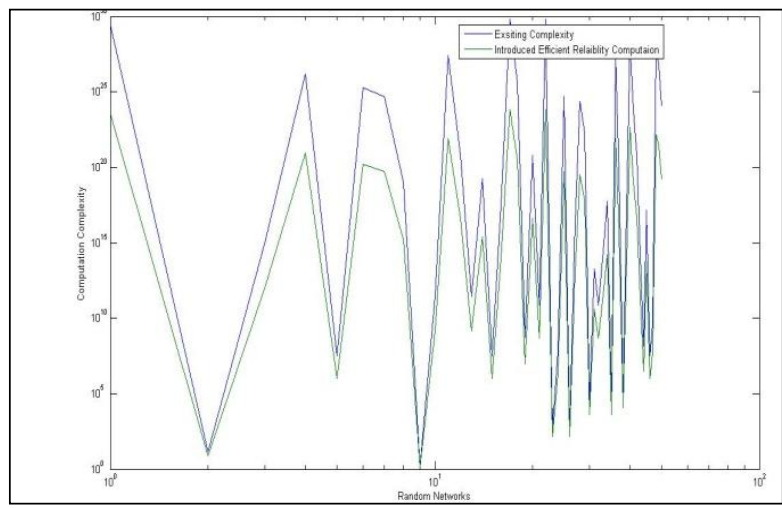

Figure 4: Critical Node Presence in Waxman Random Topologies 


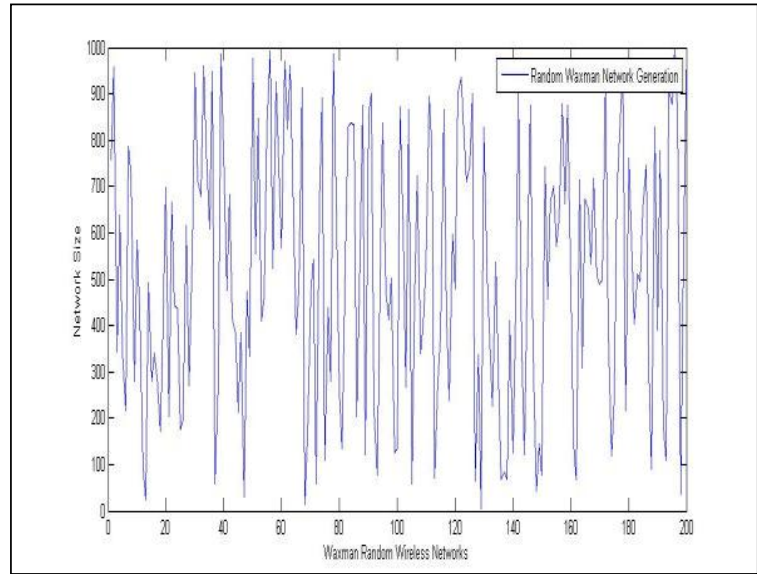

Figure 5: Simulation Results of the Proposed Algorithm in Comparison with the exisiting Specifications

\section{REFERENCES}

[1] Majid Ahmad and Durgesh Kumar Mishra. Article: A Reliability Calculations Model for Large-Scale MANETs. International Journal of Computer Applications 59(9):17-21, December 2012.

[2] Michaël Hauspie. Localized Algorithms for Detection of Critical Nodes and Links for Connectivity in Ad hoc Networks, in: "Proc. 3rd IFIP Mediterranean Ad Hoc Networking Workshop (MED-HOC-NET 2004), Bodrum,Turkey",2004.

[3] M.Jorgic, I. Stojmenovic, M. Hauspie, and D. SimplotRyl. Localized algorithms for detection of critical nodes

[4] and links for connectivity in ad hoc networks. 3rd IFIP MED-HOC-NET Workshop, 2004.

[5] A. Arulselvan, C. W. Commander, L. Elefteriadou, and P. M. Pardalos. Detecting critical nodes in sparse graphs. Comput. Oper. Res., 36(7):2193-2200, 2009.

[6] M. Duque-Anton, F. Bruyaux, P. Semal, Measuring the survivability of a network: connectivity and restconnectivity, European Transaction of Telecomunications, 11, 2, 149-159, 2000.

[7] Hung M. Dao, Silio, C.B., Jr., "Ring-network with a constrained number of consecutively-bypassed stations", Reliability, IEEE Transactions on, On page(s): 35 - 43 Volume: 47, Issue: 1, Mar 1998

[8] D. Goyal and J. Caffery, Partitioning avoidance in mobile ad hoc networks using network survivability concepts, Proc. IEEE Int. Symp. Computers and Communications ISCC, Taormina, Italy, July 2002, 553558.

[9] M. O. Ball "The complexity of network reliability computations", Networks, vol. 10, pp.153 -165 1980 [6] [9]Wei-Chang Yen, "A Simple Heuristic Algorithm for Generating All Minimal Paths", Reliability, IEEE Transactions on, On page(s): 488 - 494 Volume: 56, Issue: 3, Sept. 2007

[10] Park, K.S., Cho, B.C., "RAPID: Recursive Algorithmic PIvotal Decomposition program for complex structural reliability analysis", Reliability, IEEE Transactions on, On page(s): 50 - 53 Vol: 37, Issue: 1, 1988
[11] Cancela, H., El Khadiri, M., "The recursive variancereduction simulation algorithm for network reliability evaluation", Reliability, IEEE Transactions on, On page(s): 207 - 212 Volume: 52, Issue: 2, June 2003

[12] Jane, C.-C., Lin, J.-S., Yuan, J., "Reliability evaluation of a limited-flow network in terms of minimal cutsets", Reliability, IEEE Transactions on, On page(s): 354 - 361, 368 Volume: 42, Issue: 3, Sep 1993

[13] Hardy, G., Lucet, C., Limnios, N., "K-Terminal Network Reliability Measures With Binary Decision Diagrams", Reliability, IEEE Transactions on, On page(s): 506 - 515 Volume: 56, Issue: 3, Sept. 2007

[14] ]Fu-Min Yeh, Shyue-Kung Lu, Sy-Yen Kuo, "OBDDbased evaluation of k-terminal network reliability", Reliability, IEEE Transactions on, On page(s): 443 - 451 Volume: 51, Issue: 4, Dec 2002

[15] Hsu, S.J., Yuang, M.C., "Efficient computation of terminal-pair reliability using triangle reduction in network management", Communications, 1998. ICC 98. Conference Record. 1998 IEEE International Conference on, On page(s): 281 - 285 vol.1 Volume: 1, 7-11 Jun 1998

[16] Sy-Yen Kuo, Shyue-Kung Lu, Fu-Min Yeh, "Determining terminal-pair reliability based on edge expansion diagrams using OBDD", Reliability, IEEE Transactions on, On page(s): 234 - 246 Volume: 48, Issue: 3, Sep 1999

[17] Chin-Chia Jane; Yih-Wenn Laih; , "Computing MultiState Two-Terminal Reliability Through Critical Arc States That Interrupt Demand," Reliability, IEEE Transactions on , vol.59, no.2, pp.338-345, June 2010

[18] Bowman, M., Debray, S. K., and Peterson, L. L. 1993. Reasoning about naming systems.

[19] Ding, W. and Marchionini, G. 1997 A Study on Video Browsing Strategies. Technical Report. University of Maryland at College Park.

[20] Fröhlich, B. and Plate, J. 2000. The cubic mouse: a new device for three-dimensional input. In Proceedings of the SIGCHI Conference on Human Factors in Computing Systems

[21] Tavel, P. 2007 Modeling and Simulation Design. AK Peters Ltd.

[22] Sannella, M. J. 1994 Constraint Satisfaction and Debugging for Interactive User Interfaces. Doctoral Thesis. UMI Order Number: UMI Order No. GAX9509398., University of Washington.

[23] Forman, G. 2003. An extensive empirical study of feature selection metrics for text classification. J. Mach. Learn. Res. 3 (Mar. 2003), 1289-1305.

[24] Brown, L. D., Hua, H., and Gao, C. 2003. A widget framework for augmented interaction in SCAPE.

[25] Y.T. Yu, M.F. Lau, "A comparison of MC/DC, MUMCUT and several other coverage criteria for logical decisions", Journal of Systems and Software, 2005, in press.

[26] Spector, A. Z. 1989. Achieving application requirements. In Distributed Systems, S. Mullender. 